\section{Utilidad de los estudios de resistencia a medicamentos antituberculosos}

Pedro Valenzuela $H^{1}$
Con la aparición en nuestra época de la multirresistencia a los medicamentos antituberculosos, el empleo de pruebas de sensibilidad a estos medicamentos ha recobrado actualidad y vigencia. En general, estas pruebas cumplen dos grandes propósitos: desde un punto de vista clínico e individual, sirven para seleccionar nuevos tratamientos para pacientes sometidos a farmacoterapia múltiple que han sufrido fracaso terapéutico o resistencia a un tratamiento anterior; desde un punto de vista epidemiológico, son una herramienta de vigilancia que permite estimar periódicamente la frecuencia de resistencia en muestras de pacientes nuevos que nunca han sido tratados y de pacientes tratados previamente. Conocer la frecuencia y características de la resistencia primaria es especialmente importante para que los programas de control de la tuberculosis puedan estandarizar sus regímenes terapéuticos de inicio. Asimismo, el estudio de la resistencia adquirida o secundaria indirectamente sirve para evaluar la eficacia de la quimioterapia, elegir esquemas de tratamiento secundario estandarizados y conocer la magnitud de la fuente de resistencia primaria en una comunidad. Por último, los datos proporcionados por los estudios sobre resistencia primaria y adquirida tienen utilidad dentro de los programas de lucha antituberculosa para pronosticar el número de fracasos terapéuticos que se asociarán con la farmacoterapia estandarizada de corta duración.

\section{RESISTENCIA PRIMARIA Y ADQUIRIDA A MEDICAMENTOS ANTITUBERCULOSOS}

Se entiende por resistencia primaria a la farmacoterapia antituberculosa la que se observa en un paciente que nunca ha sido tratado. En cambio, la resistencia adquirida o secundaria se encuentra en pacientes que ya han cumplido varios meses de tratamiento o que ya fueron tratados en algún momento y se debe a la selección de cepas mutantes resistentes como consecuencia de la administración de un régimen terapéutico inadecuado. Clínicamente la resistencia secundaria se observa en dos situaciones: 1) cuando un enfermo presenta baciloscopias positivas después del cuarto mes de farmacoterapia sostenida, situación que indica fracaso terapéutico; y 2) cuando un enfermo vuelve a ser

${ }^{1}$ Instituto de Salud Pública de Chile, Santiago, Chile. 
tratado después de haber suspendido el tratamiento durante algún tiempo o de haber sufrido una recaída. Por lo general, la resistencia primaria se limita a un solo fármaco, que suele ser la estreptomicina; la secundaria, en cambio, suele involucrar a dos o más. Se habla de multirresistencia a la farmacoterapia tuberculosa cuando dos de estos fármacos son la isoniazida $(\mathrm{H})$ y la rifampicina (R).

Desde la introducción de la quimioterapia de corta duración a comienzos de los años ochenta, el programa chileno de control de la tuberculosis ha tenido la política de utilizar un único esquema terapéutico inicial estandarizado, a base de isoniazida, rifampicina, pirazinamida (Z) y estreptomizina (S), para tratar tanto a los casos nuevos de tuberculosis como a los que han recibido tratamiento en algún momento pero que lo han abandonado o han sufrido una recaída. En casos de fracaso con el régimen de corta duración se somete al paciente a un nuevo esquema de tratamiento con medicamentos distintos de los que se emplearon en el esquema inicial.

El uso de un esquema único tiene claras ventajas, pero el riesgo de fracaso es mayor en enfermos que ya han sido tratados con él que en casos nuevos de tuberculosis porque en los primeros se encuentra una proporción más alta de casos de resistencia. La resistencia primaria y adquirida difieren marcadamente en términos de su frecuencia y del tipo y número de medicamentos más comúnmente involucrados, como ya se ha señalado.

\section{ESTUDIOS SOBRE RESISTENCIA PRIMARIA Y ADQUIRIDA EN CHILE}

Chile dispone de estadísticas de resistencia primaria y secundaria desde comienzos de los años sesenta, gracias a estudios coordinados y efectuados por el antiguo Laboratorio Central y de Referencia Nacional, que ahora constituye la Sección de Micobacterias del Instituto de Salud Pública de Chile (1-3). En el período de 1963 a 1964, la frecuencia observada de resistencia primaria fue de $20 \%$ y en 1962 la resistencia secundaria llegó a 74\%. Aunque desde entonces se han realizado estudios periódicamente, no fue hasta 1971 que se introdujo una metodología de muestreo estandarizada para todo el país. Los resultados de los estudios de resistencia realizados a partir de 1971 aparecen en el cuadro 1.

Si se analizan separadamente las cifras totales de resistencia secundaria de 1985, 1988 y 1991 (4), las cuales se presentan en el cuadro 2, se observan grandes diferencias entre pacientes en tratamiento pero con signos de fracaso terapéutico (baciloscopias positivas después del cuarto mes de farmacote- rapia) y pacientes que recibieron tratamiento en el pasado pero que lo abandonaron o sufrieron una recaída. De 42 a 61\% de los enfermos en el primer grupo presentan resistencia. Entre ellos la proporción de casos de multirresistencia es muy elevada y muestra un patrón ascendente, habiendo llegado a ser de 36\% en 1991. En cambio, la resistencia en el grupo de pacientes que habían sido tratados con anterioridad tuvo una frecuencia total de 20 a $21 \%$ y la frecuencia de multirresistencia en este grupo fue de $3,7 \%$, cifra 10 veces más baja que la que corresponde al primero. Debido a esta marcada diferencia en los patrones de resistencia entre un grupo y otro, así como a lo que ella implica en términos de la elección de un tratamiento, lo más recomendable es establecer una distinción entre los dos subgrupos de enfermos con resistencia secundaria.

En el cuadro 3 se indican, para 1989 y 1991, los fármacos involucrados con mayor frecuencia en casos de resistencia primaria y adquirida y la proporción de la resistencia total representada por cada fármaco, solo o en combinación con otros. La diferencia más patente entre estos dos tipos de resistencia se observa en la proporción de casos de multirresistencia, que se aproxima a $20 \%$ en pacientes del segundo grupo y a $10 \%$ en los del primero.

\section{Estudios controlados}

Cada situación clínica conlleva un riesgo de fracaso terapéutico sustancialmente diferente. Por lo tanto, es importante conocer el riesgo de resistencia con que se asocia cada una, los fármacos involucrados en este riesgo y la proporción de la resistencia total que representa. Esto se ha demostrado en investigaciones controladas sobre quimioterapia de corta duración en la zona metropolitana de Santiago (5), en las cuales se empezó con estudios de sensibilidad inicial cuyos resultados no determinaron modificación alguna de sus respectivos protocolos terapéuticos. De 1978 a 1985, un total de 2978 enfermos de tuberculosis pulmonar con baciloscopias positivas fueron tratados según estos protocolos. Se excluyó de la muestra a los casos de abandono terapéutico, defunción, cambio de tratamiento debido a efectos secundarios adversos, y seguimiento incompleto (menos de 18 meses). Al final fueron 1970 (número representativo) los enfermos sometidos a estudios de sensibilidad inicial que completaron su seguimiento.

En los 1970 casos estudiados, la resistencia a la estreptomicina sola no implicó riesgo de fracaso terapéutico. En cambio, 14\% de los casos de resistencia a la isoniazida se asociaron con él (6). Finalmente, los casos de multirresistencia se asociaron 
CUADRO 1. Resistencia primaria y adquirida (\%) a medicamentos antituberculosos (S: estreptomicina; H: isoniazida; R: rifampicina; Z: pirazinamida). Chile, años seleccionados de 1971 a 1991

\begin{tabular}{|c|c|c|c|c|c|c|c|c|c|c|c|c|c|c|c|c|}
\hline & 1971 & 1972 & 1973 & 1975 & 1976 & 1977 & 1978 & 1979 & 1981 & 1982 & 1984 & 1985 & 1986 & 1988 & 1989 & 1991 \\
\hline \multicolumn{17}{|l|}{ Resistencia primaria } \\
\hline S & 6,8 & $\ldots$ & $\ldots$ & 4,2 & 4,3 & $\ldots$ & 3,9 & $\ldots$ & 3,5 & 4,3 & 5,3 & $\ldots$ & 6,5 & $\ldots$ & 4,7 & . \\
\hline $\mathrm{H}$ & 1,8 & $\ldots$ & $\ldots$ & 2,9 & 2,8 & . & 2,7 & $\ldots$ & 2,4 & 1,8 & 1,8 & $\ldots$ & 1,3 & $\ldots$ & 1,6 & $\ldots$ \\
\hline $\mathrm{R}$ & $\ldots$ & $\ldots$ & $\ldots$ & $\ldots$ & $\ldots$ & . & $\ldots$ & $\ldots$ & 0,2 & $\ldots$ & $\ldots$ & $\ldots$ & 0,1 & $\ldots$ & 0,2 & $\ldots$ \\
\hline Z & $\ldots$ & $\ldots$ & $\ldots$ & $\ldots$ & & $\ldots$ & $\ldots$ & $\ldots$ & . & $\ldots$ & $\ldots$ & $\ldots$ & 0,5 & $\ldots$ & . & $\ldots$ \\
\hline S-H & 2,8 & $\ldots$ & $\ldots$ & 2,9 & 4,5 & . & 2,9 & $\ldots$ & 1,6 & 2,6 & 2,7 & $\ldots$ & 1,7 & $\ldots$ & 3,0 & $\ldots$ \\
\hline $\mathrm{H}-\mathrm{R}$ & $\ldots$ & $\ldots$ & $\ldots$ & $\ldots$ & $\ldots$ & . & $\ldots$ & $\ldots$ & & $\ldots$ & & $\ldots$ & 0,2 & $\ldots$ & 0,2 & $\ldots$ \\
\hline S-H-R & $\ldots$ & $\ldots$ & $\ldots$ & $\ldots$ & $\ldots$ & . & $\ldots$ & $\ldots$ & 0,2 & $\ldots$ & 0,2 & $\ldots$ & $\cdots$ & $\ldots$ & 0,7 & . \\
\hline S-H-R-Z & $\ldots$ & $\ldots$ & $\ldots$ & $\ldots$ & $\ldots$ & $\ldots$ & $\ldots$ & $\ldots$ & $\ldots$ & $\ldots$ & 0,1 & $\ldots$ & 0,1 & $\ldots$ & $\ldots$ & $\ldots$ \\
\hline Total & 11,4 & $\ldots$ & $\ldots$ & 10,0 & 11,7 & $\ldots$ & 9,5 & $\ldots$ & 7,9 & 8,7 & 10,2 & $\ldots$ & 10,7 & $\ldots$ & 10,4 & . \\
\hline $\begin{array}{l}\text { No. de cepas estudiadas } \\
\text { Resistencia } \\
\text { adquirida }\end{array}$ & 1352 & $\ldots$ & $\ldots$ & 1208 & 925 & . & 1694 & $\ldots$ & 1737 & 1096 & 1070 & $\ldots$ & 1058 & $\ldots$ & 1167 & . \\
\hline$S$ & 14,7 & 15,7 & 13,4 & 8,7 & 5,4 & 6,9 & $\ldots$ & 3,5 & $\ldots$ & 5,7 & $\ldots$ & 6,2 & $\ldots$ & 6,0 & $\ldots$ & 8,2 \\
\hline $\mathrm{H}$ & 9,1 & 6,3 & 7,2 & 8,0 & 8,7 & 11,4 & $\ldots$ & 13,4 & $\ldots$ & 9,1 & $\ldots$ & 4,8 & $\ldots$ & 5,2 & . & 4,0 \\
\hline $\mathrm{R}$ & $\ldots$ & $\ldots$ & $\ldots$ & $\ldots$ & $\ldots$ & $\ldots$ & $\ldots$ & $\ldots$ & $\ldots$ & $\ldots$ & $\ldots$ & 0,9 & $\ldots$ & 0,6 & $\ldots$ & 1,1 \\
\hline Z & & & & $\cdots$ & & & . & $\ldots$ & $\ldots$ & . & $\ldots$ & . & $\ldots$ & 0,2 & $\ldots$ &. \\
\hline $\mathrm{T}$ & 0,5 & 0,1 & 0,3 & & & 0,1 & $\ldots$ & $\ldots$ & $\ldots$ & $\ldots$ & $\ldots$ & . & $\ldots$ & $\ldots$ & $\ldots$ & . \\
\hline S-H & 24,2 & 25,6 & 21,4 & 15,6 & 22,4 & 21,9 & $\ldots$ & 11,3 & $\ldots$ & 11,1 & $\ldots$ & 6,1 & $\ldots$ & 5,4 & $\ldots$ & 5,0 \\
\hline S-R & $\ldots$ & $\ldots$ & $\ldots$ & $\ldots$ & $\ldots$ & $\ldots$ & $\ldots$ & $\ldots$ & $\ldots$ & 0,2 & $\ldots$ & 0,9 & $\ldots$ & 1,2 & $\ldots$ & 1,3 \\
\hline $\mathrm{H}-\mathrm{R}$ & $\ldots$ & $\ldots$ & $\ldots$ & $\ldots$ & $\ldots$ & . & $\ldots$ & $\ldots$ & $\ldots$ & 1,4 & $\ldots$ & 2,5 & $\ldots$ & 1,2 & . & 0,8 \\
\hline $\mathrm{H}-\mathrm{Z}$ & $\ldots$ & $\ldots$ & $\ldots$ & $\ldots$ & $\ldots$ & . & $\ldots$ & $\ldots$ & $\ldots$ & $\ldots$ & $\ldots$ & 0,1 & $\ldots$ & 0,2 & $\ldots$ & $\ldots$ \\
\hline $\mathrm{R}-\mathrm{Z}$ & $\ldots$ & $\ldots$ & $\ldots$ & $\ldots$ & $\ldots$ & $\cdots$ & $\cdots$ & $\ldots$ & $\ldots$ & $\ldots$ & $\ldots$ & 0,1 & $\ldots$ & . & . & . \\
\hline S-H-R & . & $\ldots$ & $\ldots$ & $\ldots$ & $\ldots$ & . & $\ldots$ & $\ldots$ & $\ldots$ & 1,4 & $\ldots$ & 3,3 & $\ldots$ & 3,5 & . & 9,0 \\
\hline $\mathrm{S}-\mathrm{H}-\mathrm{Z}$ & . & $\ldots$ & $\ldots$ & $\ldots$ & $\ldots$ & . & $\ldots$ & $\ldots$ & $\ldots$ & $\ldots$ & $\ldots$ & 0,6 & $\ldots$ & . & . & 0,5 \\
\hline S-R-Z & $\cdots$ & $\ldots$ & $\ldots$ & $\cdots$ & $\ldots$ & . . & $\cdots$ & $\cdots$ & $\cdots$ & $\cdots$ & $\ldots$ & 0,1 & $\ldots$ & 0,4 & $\ldots$ & . \\
\hline $\mathrm{H}-\mathrm{R}-\mathrm{Z}$ & $\cdots$ & $\ldots$ & $\ldots$ & $\ldots$ & $\ldots$ & . & $\ldots$ & $\ldots$ & $\ldots$ & $\ldots$ & $\ldots$ & 0,1 & $\ldots$ & & . & . \\
\hline S-H-R-Z & 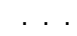 & & . & & & . & 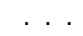 & & $\ldots$ & & $\ldots$ & 0,6 & $\ldots$ & 1,5 & $\ldots$ & 2,4 \\
\hline Total & 48,5 & 47,7 & 42,3 & 32,3 & 36,5 & 40,3 & $\ldots$ & 28,2 & $\ldots$ & 28,9 & $\ldots$ & 26,3 & $\ldots$ & 25,4 & $\ldots$ & 32,3 \\
\hline No. de cepas estudiadas & 803 & 2000 & 1582 & 588 & 334 & 720 & $\ldots$ & 550 & $\ldots$ & 636 & $\ldots$ & 690 & $\ldots$ & 522 & . & 378 \\
\hline
\end{tabular}


CUADRO 2. Resistencia adquirida (\%) a los medicamentos antituberculosos (S: estreptomicina; H: isoniazida; R: rifampicina; Z: pirazinamida) en pacientes en tratamiento pero con signos de fracaso terapéutico y en pacientes que recibieron tratamiento en el pasado y lo abandonaron. Chile, 1985, 1988 y 1991

\begin{tabular}{|c|c|c|c|}
\hline \multirow[b]{2}{*}{ Medicamento } & \multicolumn{3}{|c|}{ Resistencia adquirida } \\
\hline & 1985 & 1988 & 1991 \\
\hline \multicolumn{4}{|c|}{ Grupo en tratamiento } \\
\hline$S$ & 2,4 & 2,1 & 6,9 \\
\hline $\mathrm{H}$ & 5,9 & 8,5 & 3,0 \\
\hline $\mathrm{R}$ & 3,6 & 2,1 & 1,0 \\
\hline Z &. & 1,1 & \\
\hline $\mathrm{S}-\mathrm{H}$ & 10,7 & 6,4 & 11,9 \\
\hline S-R & 1,2 & 3,2 & 2,0 \\
\hline $\mathrm{H}-\mathrm{R}$ & 11,9 & 2,1 & 2,0 \\
\hline $\mathrm{S}-\mathrm{H}-\mathrm{R}$ & 11,9 & 13,8 & 25,7 \\
\hline S-R-Z & . & 1,1 & . \\
\hline $\mathrm{S}-\mathrm{H}-\mathrm{Z}$ & 1,2 & . & 1,0 \\
\hline S-H-R-Z & 2,4 & 2,1 & 7,9 \\
\hline Total & 54,8 & 42,5 & 61,4 \\
\hline Cepas estudiadas & 84 & 94 & 101 \\
\hline \multicolumn{4}{|c|}{ Grupo tratado previamente } \\
\hline S & 6,2 & 6,4 & 8,6 \\
\hline $\mathrm{H}$ & 3,3 & 4,0 & 4,3 \\
\hline $\mathrm{R}$ & 0,3 & . & 1,1 \\
\hline Z & 0,2 & . & 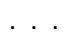 \\
\hline S-H & 5,9 & 5,4 & 2,5 \\
\hline S-R & 0,8 & 0,9 & 1,1 \\
\hline $\mathrm{H}-\mathrm{R}$ & 1,0 & 0,9 & 0,4 \\
\hline $\mathrm{H}-\mathrm{Z}$ & & 0,2 & \\
\hline $\mathrm{S}-\mathrm{H}-\mathrm{R}$ & 2,0 & 1,9 & 2,9 \\
\hline $\mathrm{S}-\mathrm{H}-\mathrm{Z}$ & 0,3 & $\ldots$ & 0,4 \\
\hline $\mathrm{H}-\mathrm{R}-\mathrm{Z}$ & 0,2 & . & . \\
\hline S-H-R-Z & 0,5 & 0,7 & 0,4 \\
\hline Total & 20,7 & 20,4 & 21,7 \\
\hline Cepas estudiadas & 606 & 428 & 277 \\
\hline
\end{tabular}

con una frecuencia estimada de fracaso terapéutico de $50 \%$ (7). Aunque los estudios no revelaron casos de resistencia a rifampicina exclusivamente, se estima que esta tiene una frecuencia semejante a la de la resistencia a isoniazida sola.

La estrecha relación entre la clase de resistencia y el riesgo de fracaso terapéutico permite usar las cifras obtenidas mediante estudios de resistencia primaria y adquirida para pronosticar, en una comunidad determinada, la fracción de la incidencia de tuberculosis que se asociará con fracaso terapéutico atribuible a resistencia medicamentosa. A manera de ejemplo se puede calcular retrospectivamente cuántos fracasos de este tipo se esperaban en Chile en 1989. Ese año se detectó resistencia primaria en $10,4 \%$ de los casos nuevos de tuberculosis (cuadro 3), lo cual implica que de cada 1000 enfermos nuevos y sin tratamiento previo, 104 tendrían
CUADRO 3. Resistencia primaria (1989) y adquirida (1991) a medicamentos antituberculosos (S: estreptomicina; $\mathrm{H}$ : isoniazida; R: rifampicina). Chile

\begin{tabular}{lcc}
\hline Medicamento & $\begin{array}{c}\text { Porcentaje } \\
\text { de casos }\end{array}$ & $\begin{array}{c}\text { Porcentaje de la } \\
\text { resistencia total }\end{array}$ \\
\hline Resistencia primaria & & \\
S sola & 4,7 & 45 \\
H o R sola o en & & \\
$\quad$ combinación & 4,8 & 46 \\
H + R (con o sin otros) & 0,9 & 9 \\
$\quad$ Total & 10,4 & \\
& & \\
Resistencia adquirida & & 40 \\
S sola & 8,6 & \\
H o R sola o en & & 10 \\
$\quad$ combinación & 9,0 & 100 \\
H + R (con o sin otros) & 4,1 & \\
$\quad$ Total & 21,7 & \\
\hline
\end{tabular}

resistencia primaria. Si a partir de esta cifra y del porcentaje de resistencia primaria observado en relación con cada fármaco se calculan y suman las cifras absolutas dadas por los porcentajes (cuadro $4)$, se obtiene un total de 12 casos $(11,5 \%)$ de fracaso terapéutico debido a resistencia primaria por cada 1000 casos nuevos de tuberculosis.

Otro ejemplo es el de 1991, año en que 21,7\% de los casos de tuberculosis tratados previamente mostraron resistencia secundaria (cuadro 3). Si se efectúa el mismo cálculo que se usó para determinar la

CUADRO 4. Fracasos terapéuticos en casos de resistencia primaria (1989) y adquirida (1991). Chile

\begin{tabular}{lrccc}
\hline Medicamento & $\%$ & No. & $\begin{array}{c}\text { Porcentaje } \\
\text { de todos } \\
\text { los fracasos }\end{array}$ & $\begin{array}{c}\text { Número } \\
\text { de } \\
\text { fracasos }\end{array}$ \\
\hline $\begin{array}{l}\text { Resistencia } \\
\text { primaria }\end{array}$ & & & & \\
$\quad(n=104)$ & & & & \\
S sola & 45 & 47 & 0 & $0^{\mathrm{a}}$ \\
H o R sola & 46 & 48 & 14 & $7^{\mathrm{a}}$ \\
H + R & 9 & 9 & 50 & $5^{\mathrm{a}}$ \\
$\quad$ Total & 100 & 104 & 64 & $12^{\mathrm{a}}$ \\
& & & & \\
Resistencia & & & & \\
$\quad \begin{array}{l}\text { adquirida } \\
\text { ( } n=217)\end{array}$ & & & & \\
S sola & 40 & 86 & 0 & $0^{\mathrm{b}}$ \\
H o R sola & 41 & 90 & 14 & $13^{\mathrm{b}}$ \\
H + R & 19 & 43 & 50 & $21^{\mathrm{b}}$ \\
Total & 100 & 219 & 64 & $34^{\mathrm{b}}$ \\
\hline
\end{tabular}

a Por 1000 casos nuevos de tuberculosis pulmonar con baciloscopia positiva.

b Por 1000 casos de tuberculosis pulmonar con baciloscopia positiva tratados previamente. 
frecuencia de resistencia primaria, se obtiene una tasa de resistencia de 217 casos por cada 1000 enfermos tuberculosos ya tratados y con antecedentes de recaída o de abandono del tratamiento. La suma de las cifras de resistencia correspondiente atribuibles a los diversos fármacos da un total de 34 fracasos terapéuticos, como indica el cuadro 4.

En 1993 se diagnosticaron aproximadamente 2200 casos de tuberculosis pulmonar bacilífera en Chile. De ellos, 86\% (1892) ocurrieron en personas sin enfermedad previa (8). Si se aplican los porcentajes anteriores, $197(10,4 \%)$ de estos 1892 casos nuevos desarrollarían resistencia primaria. Finalmente, se esperaría fracaso terapéutico en 12 de cada 1000 casos nuevos $(1,2 \%)$ (cuadro 4$)$, lo que equivale a 23 casos. Asimismo, la frecuencia de casos tratados previamente fue de $14 \%(100 \%-86 \%)$, fracción del total que equivale a 308 enfermos. Si este grupo tiene un riesgo de fracaso terapéutico de $3,4 \%$, como indica el cuadro 4 , cabe esperar que haya 10 fracasos por resistencia adquirida. Esto da un total de 33 fracasos ( 23 por resistencia primaria y 10 por resistencia secundaria) para los casos diagnosticados en 1993.

\section{Estudios de cohorte}

En Chile se estudió una cohorte de 1250 casos de tuberculosis pulmonar bacilífera para evaluar los resultados del tratamiento en el primer semestre de 1993 (8). El estudio reveló una frecuencia de fracaso terapéutico de $0,9 \%$, porcentaje que equivale a 11 casos (o a 22 cuando la cifra se proyecta al año entero) (cuadro 5). Esta cifra es menor que la calculada en la sección anterior sobre la base de los estudios controlados (33), posiblemente porque la cohorte tuvo una menor proporción de enfermos previamente tratados, especialmente con antecedentes de abandono terapéutico. La discrepancia

CUADRO 5. Resultados del tratamiento antituberculoso, según un estudio de cohorte. Chile, 1993

\begin{tabular}{|c|c|c|c|c|c|c|}
\hline \multirow{2}{*}{$\begin{array}{l}\text { Servicios } \\
\text { de salud }\end{array}$} & \multirow[b]{2}{*}{ Ingresos } & \multicolumn{5}{|c|}{ Condición al final del estudio (\%) } \\
\hline & & $\mathrm{I}^{\mathrm{a}}$ & $A^{a}$ & $\mathrm{~F}^{\mathrm{a}}$ & $D^{a}$ & $\mathrm{~T}^{\mathrm{a}}$ \\
\hline Arica & 36 & 88,9 & 8,3 & 0 & 2,8 & 0 \\
\hline lquique & 15 & 100,0 & 0 & 0 & 0 & 0 \\
\hline Antofagasta & 30 & 76,7 & 6,7 & 0 & 13,3 & 3,3 \\
\hline Atacama & 15 & 53,3 & 26,6 & 0 & 6,7 & 13,4 \\
\hline Coquimbo & 25 & 88,0 & 4,0 & 0 & 4,0 & 4,0 \\
\hline San Felipe & 31 & 93,6 & 0 & 0 & 3,2 & 3,2 \\
\hline Valparaíso & 38 & 71,0 & 2,6 & 13,2 & 13,2 & 0 \\
\hline Viña del Mar & 32 & 93,8 & 3,1 & 0 & 3,1 & 0 \\
\hline O'Higgins & 43 & 74,4 & 7,0 & 4,7 & 2,3 & 11,6 \\
\hline Maule & 47 & 74,5 & 10,6 & 2,1 & 12,8 & 0 \\
\hline Ñuble & 108 & 91,7 & 6,5 & 0 & 0,9 & 0,9 \\
\hline Concepción & 75 & 89,4 & 5,3 & 0 & 1,3 & 4,0 \\
\hline Talcahuano & 74 & 81,1 & 8,1 & 2,7 & 4,1 & 4,0 \\
\hline Bío-Bío & 49 & 4,0 & 1,2 & 0 & 3,6 & 1,2 \\
\hline Araucania & 54 & 79,6 & 9,3 & 0 & 5,6 & 5,6 \\
\hline Valdivia & 52 & 86,5 & 5,8 & 0 & 5,8 & 1,9 \\
\hline Osorno & 42 & 83,4 & 7,1 & 2,4 & 7,1 & 0 \\
\hline Llanchipal & 41 & 88,1 & 4,8 & 0 & 7,1 & 0 \\
\hline Aysen & 10 & 80,0 & 0 & 0 & 1,0 & 0 \\
\hline Magallanes & 54 & 94,4 & 1,8 & 0 & 3,8 & 0 \\
\hline Subtotal & 906 & 86,0 & 5,7 & 1,3 & 4,8 & 2,2 \\
\hline \multicolumn{7}{|l|}{$\mathrm{ZMS}^{\mathrm{b}}$} \\
\hline Norte & 51 & 80,4 & 9,8 & 0 & 3,9 & 5,9 \\
\hline Sur & 80 & 77,5 & 13,8 & 0 & 6,2 & 2,5 \\
\hline Oriental & 73 & 71,3 & 8,2 & 0 & 9,6 & 10,9 \\
\hline Occidental & 70 & 81,4 & 5,7 & 0 & 8,6 & 4,3 \\
\hline Central & 16 & 87,6 & 6,2 & 0 & 6,2 & 0 \\
\hline Sudoriental & 54 & 87,0 & 5,6 & 0 & 3,7 & 3,7 \\
\hline Subtotal & 344 & 79,2 & 8,7 & 0 & 6,8 & 5,3 \\
\hline Total & 1250 & 84,0 & 6,6 & 0,9 & 5,3 & 3,2 \\
\hline
\end{tabular}

a $\mathrm{I}=$ casos inactivos; $\mathrm{A}=$ casos de abandono del tratamiento; $\mathrm{F}=$ casos de fracaso terapéutico; $\mathrm{D}=$ defunciones; $\mathrm{T}=$ casos de traslado a otro servicio.

${ }^{b}$ Zona Metropolitana de Santiago. 
desaparece de 1994 en adelante, cuando se evalúan por separado las cohortes de enfermos con y sin tratamiento previo. También existe la posibilidad de que se hayan subestimado los fracasos debido a que algunos miembros de la cohorte se perdieron antes de completarse el seguimiento. Independientemente de ello, existe un grado aceptable de concordancia entre los resultados de las evaluaciones y la estimación basada en los estudios de resistencia.

\section{CONCLUSIONES}

La estimación de los fracasos terapéuticos esperados según la frecuencia y características de los diversos tipos de resistencia a los fármacos antituberculosos no es un mero ejercicio aritmético y probabilístico. Es un cálculo fundamental que ayuda a los directores de programas antituberculosos a planificar la adquisición y el abastecimiento de medicamentos de reserva para los casos que necesitan un cambio de tratamiento, así como las consultas a especialistas, hospitalizaciones, tratamientos de larga duración y otros detalles operacionales asociados con los fracasos estimados. Por otra parte, la cuantificación de los fracasos, separándolos según sus antecedentes terapéuticos, también sirve de base para aplicar esquemas de tratamiento diferentes a distintos tipos de enfermos, como preconiza la OMS (9). Finalmente, la posibilidad de usar para fines predictivos la magnitud de la resistencia primaria y secundaria a los medicamentos antituberculosos debe motivar a los países a intensificar la vigilancia epidemiológica y a calcular sus propias estadísticas sobre la resistencia dentro del territorio nacional y sus subdivisiones.

\section{REFERENCIAS}

1. Valenzuela P, Lepe R, Ponce de León L, et al. Evolución de la resistencia de $M$. tuberculosis en Chile. Rev Med Chile 1973; 101:30.

2. Valenzuela MT, Scheel G, Ponce J, et al. Resistencia a drogas antituberculosas en Chile. Rev Med Chile 1984;112:76-80.

3. Guzmán AM, García P, Valenzuela MT, et al. Resistencia adquirida del M. tuberculosis a las drogas antibacilares en Chile, 1988 y 1991. Rev Med Chile 1993;121:12691273.
4. Guzmán AM, Valenzuela P. Enfermos de tuberculosis antes tratados iun esquema terapéutico especial? Rev Chil Enf Resp 1991;7:164-167.

5. Farga V, Valenzuela P, Valenzuela MT, et al. Una década de quimioterapia antituberculosa abreviada en Chile. Rev Chil Enf Resp 1991;7:94-106.

6. Valenzuela MT, et al. Respuesta a la quimioterapia de corta duración en enfermos con resistencia inicial a los medicamentos antituberculosos. Bol Oficina Sanit Panam 1988;104:365-373.
7. World Health Organization. Report of a WHO meeting. Geneva: WHO; octubre de 1990. (Documento WHO/TB/91-160).

8. Chile, Ministerio de Salud. Informe de evaluación del programa de control de la tuberculosis, 1993. Santiago: MS; 1994.

9. World Health Organization. Guidelines for tuberculosis treatment in adults and children in National Tuberculosis Programmes. Geneva: WHO; 1991. (Documento WHO/ TUB/91-161). 